\title{
JUSTICE SOCIALE ET ÉQUITÉ
}

\section{NOTE DE SYNTHĖSE}

SUR LES RÉSULTATS FRANÇAIS

DE LA 9E ÉDITION DE L'ENQUÊTE SOCIALE EUROPÉENNE

\section{Cyril JAYET}

Maître de conférences en Sociologie,

Sorbonne Université

GEMASS (CNRS, Sorbonne Université)

\section{Gemass}

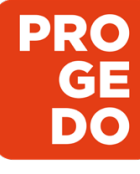

.

ASTRUCTURE

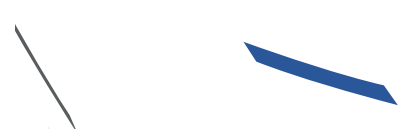


Dans l'espace médiatique et politique, le thème des inégalités a pris ces dernières années une ampleur considérable, ce dont témoigne notamment le très grand succès rencontré par louvrage de Thomas Piketty Le Capital

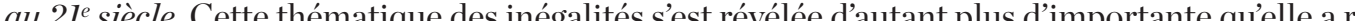
contexte de orrande défiance à légard du personnel politique. Cette dernière a été particulièrement manifeste lors du mouvement des gilets jaunes, l'un des plus longs mouvements sociaux de l'histoire récente.

L’intensité de cette remise en cause du système économique comme des élites politiques et du système partisan nous rappelle l'importance de mieux comprendre la formation des jugements politiques et économiques, ceuxci contribuant à l'acceptation du système en place, ou au contraire à sa contestation. Les travaux portant sur les sentiments de justice des Français montrent que ces derniers considèrent vivre dans une sociététrop inégalitaire sociale et équité de l'enquête ESS, administré entre octobre 2018 et mars 2019 a l 1 données inédites permettant d'examiner si ce constat partagé d'une injustice de la société se retrouve lorsque les individus jugent de leur situation personnelle et de ses différentes dimensions : niveaux de richesse, trajectoire scolaire ou encore capacité à trouver un emploi. Ce module permet ainsi d'examiner si la défiance politique s’enracine dans des jugements négatifs sur sa propre trajectoire personnelle et les injustices qu’elle comprend.

\section{UNE INJUSTICE PARTAGÉE}

Le nouveau module de l'enquête ESS a l'avantage de poser d'une part des questions amenant les enquêté.e.s à juger de l'inégalité et de l'équité de la société française, et d'autre part des questions très similaires portant sur la situation et la trajectoire personnelles de l'enquêté.e. On examinera d'abord si ces deux types de jugement diffèrent sensiblement dans l'ensemble de la population, puis si ces différences varient elles-mêmes selon les groupes sociaux 1 .

L'égalité des richesses et la rémunération personnelle

Le graphique 1 présente les réponses des enquêtés à deux questions, la première portant sur le caractère juste ou non des différences de richesse en France et la seconde sur le sentiment de justice à l'égard de sa propre rémunération. Le premier constat qui se dégage est celui d'un accord très majoritaire sur l'injustice des différences de richesses en France. Ainsi, seule une petite minorité, $9 \%$, juge que ces différences sont justes. Les Français sont au contraire $62 \%$ à juger qu'elles sont trop grandes. Il faut noter également qưune minorité importante les jugent trop petites (29\%).

Graphique 1 - Inégalité de richesse et jugement à légard de sa rémunération

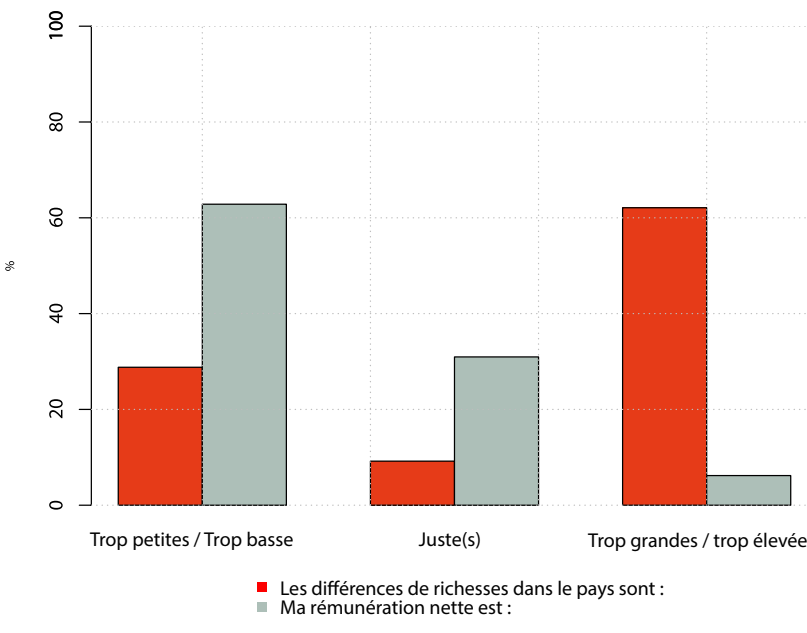

Note: Dans le questionnaire, l'échelle pour le pays varie de - 4 (extrêmement injustes, trop petites) à +4 (extrêmement injuste, trop grandes) en passant par 0 (juste). Pour la rémunération nette, l'échelle varie de - 4 (extrêmement injustes, trop basse) à +4 (extrêmement injuste. trop élevée) en passant par 0 ( juste). Nous avons recodé dans les deux cas en trois catégories.
Un sentiment majoritaire d'injustice se retrouve lorsque l'individu juge de sa propre rémuneration : $62 \%$ des enquêtés considèrent leur rémunération comme injustement basse. Les résultats font néanmoins apparâtre une importante différence Comme soulioné plus hant, seuls $9 \%$ des enquêtés cons fóraient les différences de richeses comme justes ils sont en revanche $31 \%$ à trouver leur salaire juste. En outre les données mettent en évidence que si les différences de richesses en France apparaissent trop élevées à la grande majoritédes enquêtés (62\%), il est rare de se considérer soi-même comme trop favorisé: les enquêtés ne sont que $6 \%$ à trouver leur rémunération trop élevée. Ce fort sentiment d'injustice salariale n’est d'ailleurs pas une spécificité française. On le retrouve dans l'ensemble des pays couverts par l'ESS, quỉls soient bien ou mal lotis du point de vue de leur niveau de richesse mesuré par le PIB.

L'égalité des chances

Des résultats assez similaires peuvent se retrouver si l'on examine l'égalité des chances. Celle-ci peut être appréhendée à partir de deux questions, l'une portant sur l'accès au niveau d'étude que l'on souhaite, la seconde sur l'accès à l'emploi que l'on souhaite. Là encore, les jugements sur la société sont plutôt négatifs (graphique 2) : les individus jugent majoritairement que tout le monde n’a pas une chance équitable d'atteindre l'emploi (57\%) ou le niveau d'étude qu'il souhaite ( $47 \%)$

\section{Graphique 2 - Égalité des chances face à l'éducation et l'emplo}

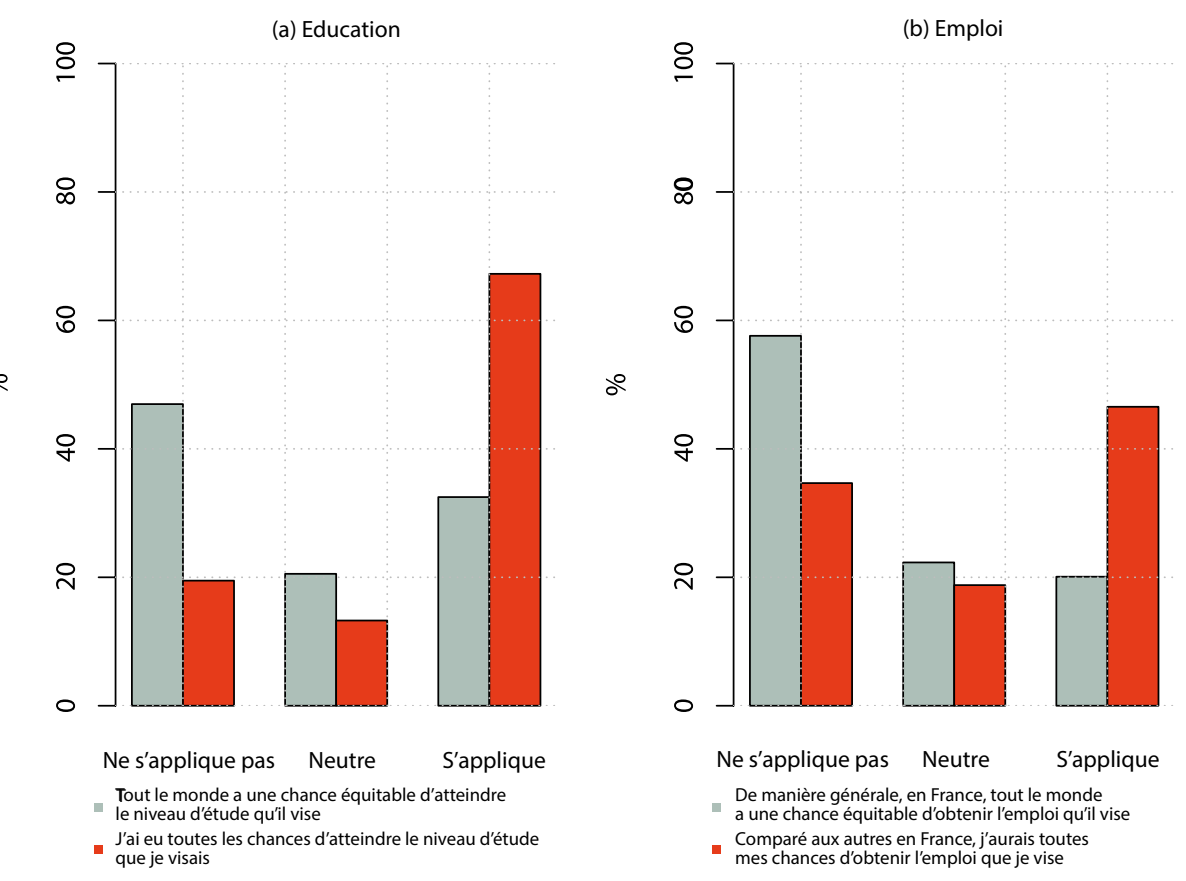
Note: : Dans le questionnaire, l'échelle varie de 0 (ne s’applique pas du tout) à 10 ( (’applique tout à fait) en passant par 0 (juste)
Nous avons recodé dans les deux cas en trois catégories en considérant 5 comme la réponse neutre.

Ces jugements sont cependant moins négatifs lorsque les individus évaluent leur propre situation, suivant en cela une tendance bien connue à l'optimisme sur soi mais au pessimisme sur la société. Sur le niveau d'étude, les répondants considèrent très majoritairement (68\%) qưils ont eu la même chance que les autres d'atteindre leur niveau d'étude. Sur 'emploi, 48\% des enquêtés répondent que s'ils cherchaient un emploi, ils auraient la même chance que les autres de trouver l'emploi qu'ils visent, réponse là encore majoritaire.

Le tableau d'ensemble que révèlent ces différents résultats est donc plutôt celui d'une critique assez majoritaire du système social. Le jugement sur la situation personnelle est plus contrastée: si la grande majorité des enquêtés jugent leur salaire injustement bas, ils jugent aussi majoritairement avoir eu les mêmes chances que les autres d’avoir obtenu leur niveau d'étude ou qu'ils auraient les mêmes chances que les autres de trouver un emploi s'ils en cherchaient un. On observe ainsi un phénomène similaire à l'effet troisième personne en étude des médias. Ce dernier désigne le fait que la majorité des individus sont convaincus que les médias influencent les opinions des autres mais pas les leurs Ici la majorité des gens considèrent cüils ont eu les mêmes chances que les autres, mais que tout le monde n’a pas les mêmes chances. Ce dernier résultat semble contredire lidée selon laquelle la critique de l'injustice du système s'enracinerait dans une critique des injustices que l'on subit soi-même. 


\section{STRATIFICATION SOCIALE DES JUGEMENTS}

Nous pouvons maintenant essayer de mieux comprendre ces jugements sur les inégalités en examinant leur distribution dans différents groupes socioprofessionnels ${ }^{2}$. Il s'agira ainsi de comprendre s'ils s'enracinent dans des différences de conditions de vie.

Inégalités de richesses et de rémunération

D'un point de vue utilitariste, on pourrait faire l'hypothèse d'une critique des inégalités plus faible chez les plus favorisés puisqu’ils sont bénéficiaires du système. Cette théorie simple décrit néanmoins mal les données. $\mathrm{Ce}$ sont en effet les cadres qui répondent le plus souvent que les inégalités sont trop grandes : c'est le cas de $68 \%$ des cadres contre $62 \%$ des employés, $58 \%$ des ouvriers et $58 \%$ des artisans et commerçants. Au contraire, ce sont les employés (30\%) et les ouvriers (33\%) qui répondent le plus qưelles sont trop petites (contre 23\% des cadres). Cet effet du groupe socioprofessionnel est étroitement lié à l'influence du niveau de diplôme, les individus les plus diplômés étant généralement les plus critiques des inégalités.

\section{Tableau 1 - Groupe socioprofessionnel et jugement sur les inégalités}

\begin{tabular}{lcccc} 
& \multicolumn{4}{c}{ Les différences de richesses dans le pays sont } \\
\cline { 2 - 5 } & Injuste, trop petites & Juste & $\begin{array}{c}\text { Injuste, trop } \\
\text { grandes }\end{array}$ & Total \\
\hline Artisan, commerçant & 30.2 & 12.1 & 57.8 & 100 \\
Cadre et prof. Int. & 23.1 & 9.3 & 67.6 & 100 \\
Prof. Inter. & 29.1 & 9.4 & 61.4 & 100 \\
Ouvrier & 32.6 & 9.3 & 58.0 & 100 \\
Employé & 30.0 & 7.6 & 62.4 & 100 \\
Ensemble & 28.7 & 9.2 & 62.1 & 100 \\
\hline
\end{tabular}

Note: Dans le questionnaire, l'échelle pour le pays varie de -4 (extrêmement injustes, trop petites) à 4 (extrêmement injuste, trop grandes) en passant par 0 (juste). Nous avons recodé cas en trois catégogries.

Si ce ne sont donc pas les moins favorisés qui trouvent le plus souvent que les inégalités sont trop grandes, ce sont bien eux qui jugent le plus souvent leur rémunération comme trop basse (tableau 2). Ainsi 70\% des ouvriers, $64 \%$ des employés et $69 \%$ des artisans et commerçants trouvent leur rémunération trop basse, contre $53 \%$ des cadres. Les cadres sont au contraire $41 \%$ à trouver leur rémunération juste contre seulement $23 \%$ des ouvriers, $29 \%$ des employés et $25 \%$ des artisans et commerçants.

\section{Tableau 2 - Groupe socioprofessionnel et jugement sur sa rémunération}

\begin{tabular}{lcccc}
\cline { 2 - 5 } & \multicolumn{4}{c}{ Ma rémunération nette est } \\
\hline Artisan, commerçant & Injuste, trop basse & Juste & Injuste, trop haute & Total \\
Cadre & 69.3 & 25.4 & 5.3 & 100 \\
Prof. inter. & 52.5 & 41.4 & 6.1 & 100 \\
Employé & 59.0 & 35.1 & 5.9 & 100 \\
Ouvrier & 64.3 & 28.8 & 7.0 & 100 \\
Ensemble & 70.4 & 22.9 & 6.8 & 100 \\
\hline
\end{tabular}

Note: Dans le questionnaire, l'échelle varie de -4 (extrêmement injustes, trop basse) à +4 (extrêmement injuste, trop élevée)

en passant par 0 (juste). Nous avons recodé en trois catégories.
Les jugements sur les inégalités n’opposent donc pas de manière simple les groupes socioprofessionnels ou pas d'une manière qui transposerait directement à la société le jugement sur sa situation personnelle. Ainsi, les

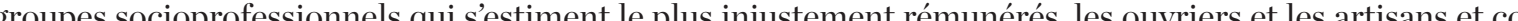
merçants, ne sont pas ceux qui trouvent le plus souvent les inégalités de richesses trop grandes, ils ont même plus souvent tendance que les cadres à les trouver trop basses.

Égalité des chances et groupe socioprofessionnel

Les tableaux 3a et 3b présentent la relation entre l'appartenance à un groupe socioprofessionnel et les jugements sur l'équité en France. Là encore, ce ne sont pas les plus favorisés par le système qui juơent le système plus juste. Ce sont ainsi les artisans, les commerçants et les ouvriers qui répondent le plus souvent que tout le monde a une chance équitable d’atteindre le niveau d'étude ou l'emploi qu'il vise. A propos de léducation, ils sont ainsi respectivement $41 \%$ et $38 \%$ à donner cette réponse contre $28 \%$ des employés, $30 \%$ des professions intermédiaires et 33\% des cadres. Les réponses concernant l'emploi sont assez proches, les ouvriers (55\%), les artisans et les commerçant (53\%) répondant un peu moins souvent que les cadres (60\%) que tout le monde n’a pas une chance équitabled nalisation socia nalisation socialement différenciée des normes dégalité des chances. Les ouvriers exprimeraient ainsi ici une forme de croyance dans la méritocratie, tandis que les cadres, plus diplômés, pourraient davantage relativiser l'idéologie méritocratique.

\section{Tableau 3 - PCS, chance d'atteindre niveau éducation}

(a) Tout le monde a une chance équitable d'atteindre le niveau d'études qưil vise

\begin{tabular}{lcccc} 
& Ne s'applique pas & Réponse neutre & Sapplique & Total \\
\hline Artisan, commerçant & 38.1 & 21.2 & 40.7 & 100 \\
Cadre & 49.9 & 16.8 & 33.3 & 100 \\
Prof. inter. & 49.4 & 20.6 & 30.0 & 100 \\
Employé & 48.7 & 23.7 & 27.6 & 100 \\
Ouvrier & 40.0 & 22.3 & 37.7 & 100 \\
Ensemble & 46.7 & 20.8 & 32.5 & 100 \\
\hline
\end{tabular}

(b) De manière générale, en France, tout le monde a une chance équitable d’obtenir l'emploi quiil vise

\begin{tabular}{lcccc} 
& Ne s’applique pas & Réponse neutre & Sapplique & Total \\
\hline Artisan, commerçant & 52.5 & 23.7 & 23.7 & 100 \\
Cadre & 60.1 & 19.1 & 20.9 & 100 \\
Prof. inter. & 55.6 & 23.9 & 20.5 & 100 \\
Employé & 59.3 & 24.0 & 16.7 & 100 \\
Ouvrier & 55.1 & 20.5 & 24.3 & 100 \\
Ensemble & 57.5 & 22.0 & 20.5 & 100 \\
\hline
\end{tabular}

Note: Dans le questionnaire, l'échelle varie de 0 (ne s'applique pas du tout) à 10 (s'applique tout à fait) en passant par 0 (juste). Nous avons recodé dans les deux cas en trois catégories en considérant 5 comme la réponse neutre.

La relation entre groupe socioprofessionnel et jugement sur léquité va là encore dans le sens attendu (Tableaux 4a et 4b). Les ouvriers et les employés estiment ainsi beaucoup moins souvent qưils ont eu la même chance que les autres d'obtenir le niveau d'étude qu'ils visaient ou qu'ils pourraient avoir l'emploi qu'ils souhaiteraient. La différence est particulièrement forte sur le niveau d'étude : $87 \%$ des cadres jugent qưils ont eu les mêmes chances que les autres, contre seulement $53 \%$ des ouvriers ou $58 \%$ des employés. 
Tableau 4 - PCS, chance d'atteindre niveau éducation

(a) J'ai eu toutes les chances d'atteindre le niveau d'étude que je visais.

\begin{tabular}{lcccc}
\cline { 2 - 5 } & Ne s'applique pas & Réponse neutre & S'applique & Total \\
\hline Artisan, commerçant & 17.1 & 16.2 & 66.7 & 100 \\
Cadre & 7.4 & 5.9 & 86.6 & 100 \\
Prof. inter. & 11.5 & 9.4 & 79.1 & 100 \\
Employé & 24.6 & 17.4 & 58.0 & 100 \\
Ouvrier & 29.1 & 17.7 & 53.2 & 100 \\
Ensemble & 17.9 & 12.8 & 69.3 & 100 \\
\hline
\end{tabular}

(b) Comparé aux autres en France, jaurais toutes mes chances d'obtenir l'emploi que je vise

\begin{tabular}{lcccc} 
& Ne s'applique pas & Réponse neutre & S'applique & Total \\
\hline Artisan, commerçant & 28.6 & 22.3 & 49.1 & 100 \\
Cadre & 22.2 & 12.0 & 65.8 & 100 \\
Prof. inter. & 31.2 & 16.9 & 51.9 & 100 \\
Employé & 42.4 & 23.1 & 34.4 & 100 \\
Ouvrier & 39.9 & 20.5 & 39.5 & 100 \\
Ensemble & 33.2 & 18.4 & 48.4 & 100 \\
\hline
\end{tabular}

Note: Dans le questionnaire, léchelle varie de 0 (ne sápplique pas du tout) à 10 ('’applique tout à fait) en passant par 0 (juste)
Nous avons recodé dans les deux cas en trois catégories en considérant 5 comme la réponse neutre.

Les résultats sur l'équité confirment donc ceux obtenus sur l'égalité : une position moins favorisée suscite un sentiment d' injustice à l'egard de sa situation personnelle, mais pas a l'egard du fonctionnement d'ensemble du système social. Ainsi, quand bien même les ouvriers et les employés se jugent moins favorisés que les cadres, ce sont ces derniers qui jugent la société comme injuste.

\section{SENTIMENT D'INIQUITÉ ET DÉFIANCE VIS-À-VIS DE L'ACTION DU GOUVERNEMENT}

Si les sentiments d'injustice à légard de sa situation personnelle sont donc moins répandus que ceux à l'égard de la société, ils sont socialement très différenciés. Ils pourraient ainsi expliquer une part de la défiance politique et des clivages sociaux qui la sous-tendent.

Le graphique 4 présente la défiance à l'égard de l'action du gouvernement dans les différents groupes socioprofessionnels. Il montre quà l'exception des cadres, la grande majorité des enquêtés jugent que le gouvernement ne prend pas en compte les intérêts de tous. Cette défiance à l'égard du gouvernement est donc à la fois très répandu et socialement différenciés : elle est très élevée chez les artisans et commerçants $(70 \%)$ et, chez les salariés, elle est maximale chez les ouvriers. Dans l'ensemble, les enquêtés ne sont que $6 \%$ à répondre que le gouvernement prend beaucoup ou complètement en compte les intérêts de tous les citoyens contre $61 \%$ qui répondent pas du tout ou très peu.
Graphique 4 - Le gouvernement français prend en compte les intérêts de tous les citoyens?

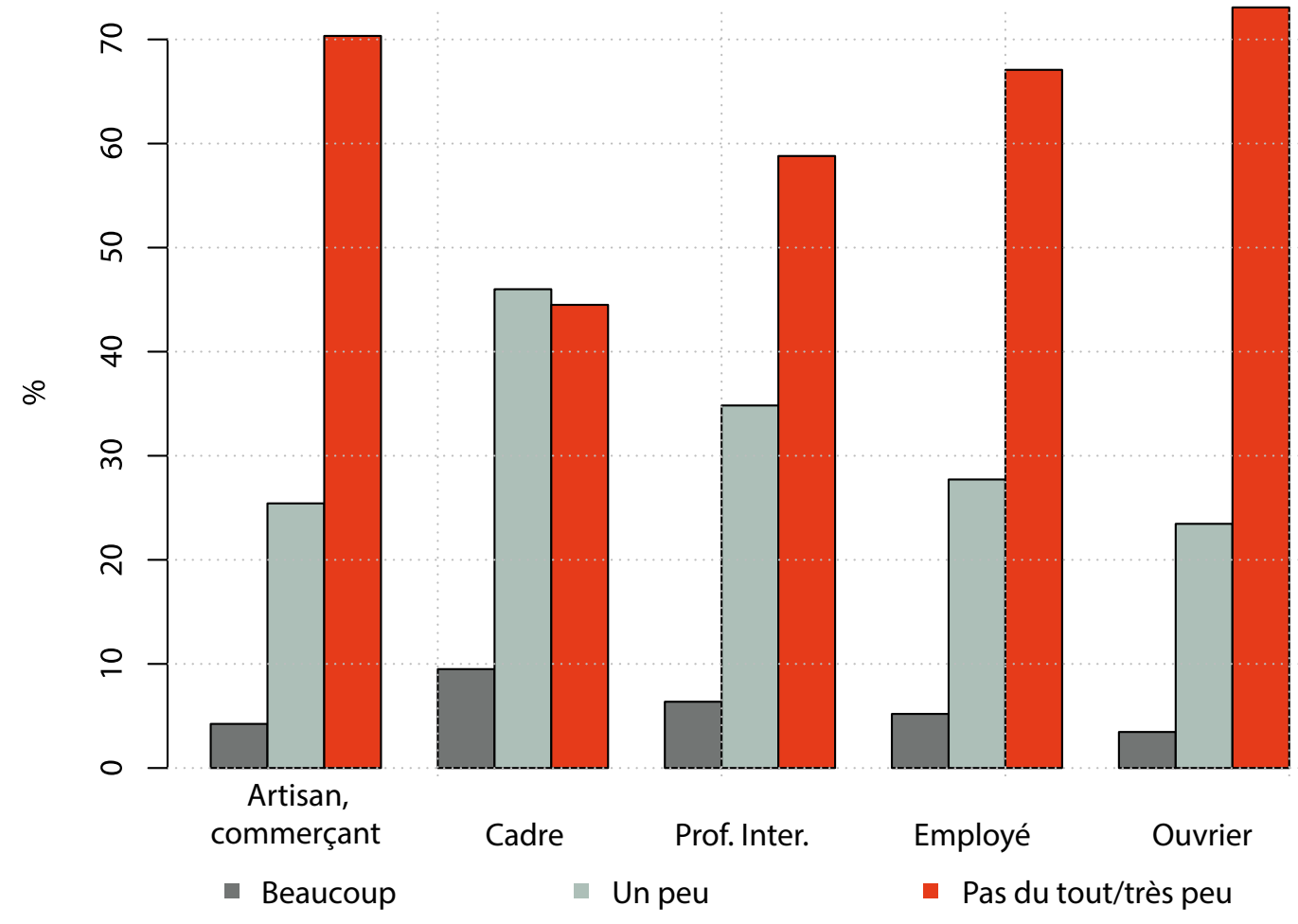

Pour comprendre si cette défiance à l'égard du gouvernement peut s'expliquer par des jugements sur l'injustice de sa situation ou de sa trajectoire, nous avons estimé plusieurs modèles de régression logistique prenant cette défiance pour variable à expliquer. Les différents modèles montrent tout d'abord que les individus sont d'autant moins critique à l'égard du gouvernement qưils se situent politiquement au centre 3 et qu’ils s'intéressent à la politique. Le premier modèle estime l'effet du groupe socioprofessionnel et montre que, à âge, sexe, positionnement et intérêt politique constants, tous les groupes socioprofessionnels ont moins de chances que les cadres de juger que le gouvern de l'individu sur sa rémunération, le troisième sur ses chances d'atteindre son niveau d'étude et le quatrième comprend ces deux variables. On observe alors que la différence entre les cadres et les autres groupes socioprofessionnels se réduit nettement. Le coefficient mesurant l'effet de la modalité « Ouvrier » diminue ainsi de 9\% en introduisant le jugement sur la rémunération, de $15 \%$ sur la trajectoire scolaire et de $17 \%$ en tenant compte des deux. Ce sont ainsi les jugements sur la trajectoire scolaire personnelle qui paraissent le mieux expliquer 列 parant les cadres avec les autres groupes socioprofessionnels. Pour les employés, cette réduction de lécart avec les cadres est particulièrement importante : le coefficient diminue de $16 \%$ en introduisant le jugement sur la rémunération, de $34 \%$ en introduisant le jugement sur la trajectoire scolaire et de $42 \%$ quand on introduit les deux variables. C'est ainsi près de la moitié des différences de jugement entre les cadres et les employés que l'on peut expliquer par un sentiment d'injustice à l'égard de sa rémunération et de sa trajectoire scolaire. C'est aussi cette dernière variable qui se révèle la plus importante pour expliquer les différences de jusement entre les deux groupes ${ }^{4}$. 


\section{A propos de liESS}

Le gouvernement ne prend pas en compte l'intérêt de tous

PCS (référence. Cadre)

Artisan, commerçant

Prof. Inter.

Employé

Ouvrier

Age

Femme

Droite (vs. gauche, 0-10)

Droite`2

$\begin{array}{lccc}1.33^{* * * *} & 1.17^{* * *} & 1.19^{* * * *} & 1.12^{* * *} \\ 0.61^{* * *} & 0.55^{* * *} & 0.51^{* * *} & 0.48^{* *} \\ 0.92^{* * *} & 0.77^{* * * *} & 0.61^{* * *} & 0.53^{* * *} \\ 1.30^{* * *} & 1.18^{* * *} & 1.11^{* * *} & 1.07^{* * *} \\ 0.001 & 0.002 & -0.0004 & -0.0001 \\ 0.39^{* * *} & 0.40^{* * * *} & 0.44^{* * *} & 0.45^{* * *} \\ -0.57^{* * *} & -0.59^{* * * *} & -0.59^{* * * *} & -0.60^{* * *} \\ 0.04^{* * *} & 0.04^{* * *} & 0.04^{* * *} & 0.05^{* * *}\end{array}$

Intérêt politique (réf. Pas du tout)

Peu

Assez

Beaucoup

$\begin{array}{llll}-0.73^{* * *} & -0.74^{* * *} & -0.69^{* * * *} & -0.71^{* * *} \\ -0.97^{* * *} & -0.92^{* * *} & -0.91^{* * *} & -0.87^{* * *} \\ -0.91 * * & -0.98 * & -0.88^{* * *} & -0.93^{* * *}\end{array}$

$-0.91^{* * *}-0.98^{* * *} \quad-0.88^{* * *} \quad-0.93^{* * *}$

Ma rémunération est injustemen haute (vs. juste et injustement basse)

$-0.19^{* * * *}$

J'ai eu toutes les chances d'atteindre le niveau d'étude que je visais (0-10)

\begin{tabular}{lcccc} 
Constante & $1.94^{* * * *}$ & $2.75^{* * * *}$ & $3.10^{* * * *}$ & $3.55^{* * * *}$ \\
\hline Observations & 1353 & 1300 & 1316 & 1266
\end{tabular}

"p $<0.1 ; * * \mathrm{p}<0.05 ;{ }^{* * *} \mathrm{p}<0.01$

Note: Coefficients issus de modèles de régression logistique dichotomiques. La variable dépendante a été transformée en variable
binaire opposant les réponses «Beaucoup », «Complétement» et « un peu » aux réponses «très peu» et «pas du tout»

CONCLUSION

Le module Justice sociale et équité de l'enquête ESS sur la France met en lumière plusieurs résultats importants. Tout d'abord, le constat partagé en 2019 par les Français d'une injustice du système actuel dans des domaines aussi différents que les différences de richesse, l'accès au niveau d'étude que l’on souhaite ou encore la prise en compte des intérêts de tous par le gouvernement. Ensuite, ce constat d'injustice se révèle plus important lorsque les individus jugent de l'état du pays que lorsqu'ils jugent de leur propre situation. Si les Français trouvent majoritairement leur salaire trop bas, ils jugent également majoritairement qưils ont eu les mêmes chances que les autres d'atteindre le niveau d'étude qu'ils visaient ou encore qưils auraient les mêmes chances que les autres de trouver un emploi s'ils en cherchaient un. Ces derniers jugements diffèrent néanmoins fortement selon le groupe socioprofessionnel. Les groupes les plus défavorisés considèrent ainsi plus souvent avoir eu moins de chances que les autres. Ce sentiment d'iniquité est fortement associéà une défiance vis-à-vis de la capacité du gouvernement à prendre en compte l'intérêt général. En outre, il explique une part importante de cette défiance dans les groupes socioprofessionnels les moins favorisés. Un sentiment d'iniquité et d'injustice se trouve ainsi bien à la racine des divisions sociales et politiques de jugement sur l'action du gouvernement.

Référence bibliographique:

GALLAND, Olivier et FORSÉ, Michel. Les Françaisface aux inégalités et à lajustice sociale. Armand Colin, 2011.
L'ESS est une enquête universitaire menée partout en Europe depuis 2002. L'étude mesure les attitudes, les croyances et le comportement de diverses populations dans plus de 30 pays.

Les jeux de données contiennent les résultats de 425000 entretiens réalisés tous les deux ans auprès de nouveaux échantillons de population.

L'Enquête Sociale Européenne est devenue un Consortium Européen d'Infrastructure de Recherches (ERIC) en 2013.
Thématiques de l'ESS :

- Confiance dans les institutions

- Participation politique

- Valeurs socio-politiques

- Valeurs morales et sociales

- Capital social

- Intégration et exclusion sociale

- Identités religieuses, ethniques et nationales

- Santé, bien-être et sécurité

- Éducation et emploi
- Composition démographique
- Situation financière

- Composition du ménage

- Bien-être subjectif

- Confiance dans la justice

- Perceptions et expériences des âges de la vie - Citoyenneté, participation et démocratie - Immigration

- Famille, travail et bien-être

- Moralité économique, justice sociale et équité

- Organisation du déroulement de la vie

- Changement climatique et énergie

- Échelle des valeurs humaines
27 pays ont participé à la 9e édition de l'ESS, menée en 2018/2019.

Membres : Allemagne, Autriche, Belgique, Bulgarie, Chypre, Croatie, Estonie, Finlande, France,

Hongrie, Irlande, Italie, Lettonie, Lituanie, Norvège, Pays-Bas, Pologne, Portugal, République Tchèque, Royaume-Uni, Slovaquie, Slovénie et Suède Pays observateur : Suisse Autres pays participants : Espagne, Monténégro et Serbie.

Groupes consultatifs de l'Assemblée Générale de l'ESS ERIC : Methods Advisory Board (Conseil Consultatif Méthodes - MAB), Scientific Advisory Board (Conseil Consultatif Scientifique - SAB) et le Comité Finances (FINCOM). Le siège de l'ESS ERIC est situé à la City Université de Londres. L'équipe scientifique centrale de l'ESS ERIC comprend : le GESIS - Institut de Sciences Sociales de Leibniz (Allemagne) ; 'Université Catholique de Louvain (Belgique) ; le NSD - Centre Norvégien pour les données de recherche (Norvège) ; le SCP - Institut Néerlandais de Recherche en Sciences Sociales (Pays-Bas) ; l'Université Pompeu Fabra (Espagne) ; l'Université d'Essex (Royaume-Uni) et I'Université de Ljubljana (Slovénie).

Le Forum des Coordinateurs Nationaux (NC) regroupe des équipes nationales originaires de tous les pays participants.

europeansocialsurvey.org

esswellbeingmatters.org

twitter.com/ESS_Survey

facebook.com/EuropeanSocialSurvey

linkedin.com/european-social-survey

youtube.com/EuropeanSocialSurveyERIC

mars 2021

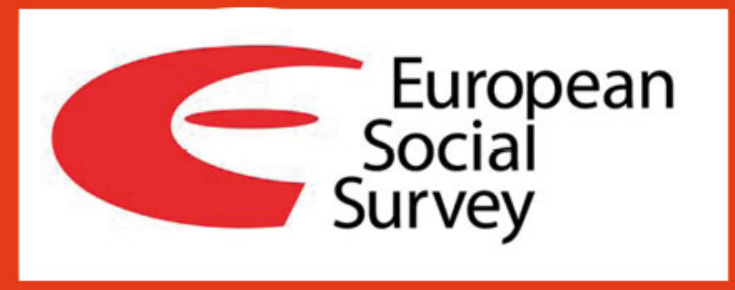

